\title{
A Common Fixed Point Theorem for Generalised F-Kannan Mapping in Metric Space with Applications
}

\author{
Lucas Wangwe $(\mathbb{D})$ and Santosh Kumar $(\mathbb{D}$ \\ Department of Mathematics, College of Natural and Applied Sciences, University of Dar es Salaam, Tanzania \\ Correspondence should be addressed to Santosh Kumar; drsengar2002@gmail.com
}

Received 7 December 2020; Accepted 30 March 2021; Published 17 April 2021

Academic Editor: Alberto Fiorenza

Copyright (c) 2021 Lucas Wangwe and Santosh Kumar. This is an open access article distributed under the Creative Commons Attribution License, which permits unrestricted use, distribution, and reproduction in any medium, provided the original work is properly cited.

This paper is aimed at proving a common fixed point theorem for $F$-Kannan mappings in metric spaces with an application to integral equations. The main result of the paper will extend and generalise the recent existing fixed point results in the literature. We also provided illustrative examples and some applications to integral equation, nonlinear fractional differential equation, and ordinary differential equation for damped forced oscillations to support the results.

\section{Introduction and Preliminaries}

In 1922, Banach [1] established a fixed point theorem in a metric space which states that if $X$ is a complete metric space and $T: X \longrightarrow X$ is a contraction map, i.e., $d(T x, T y)$ $\leq k d(x, y)$ for all $x, y \in X$ and $k \in[0,1)$, then $T$ has a unique fixed point or $T x=x$ has a unique solution. Since then, researchers are finding the ways to determine the fixed points of the maps by changing one or more conditions such as contractive condition, continuity of the maps, and completeness of the space etc. Kannan [2] gave an alternative contractive condition which was different from the Banach contraction condition. In 1968, Kannan [2] used this new contractive condition and proved the following theorem for selfmappings in complete metric spaces as a result of a generalisation of the Banach fixed point theorem.

Theorem 1 (see [2]). Let $(X, d)$ be a complete metric space and a self-mapping $T: X \longrightarrow X$ be a mapping such that

$$
d(T x, T y) \leq k\{d(x, T x)+d(y, T y)\}
$$

for all $x, y \in X$ and $0 \leq k \leq 1 / 2$. Then, $T$ has a unique fixed point $z \in X$ and for any $x \in X$ the sequence of iterate $\left\{T^{n} x\right\}$ converges to $z$.
An equivalent form of (1),

$$
d(T x, T y) \leq \frac{k}{2}\{d(x, T x)+d(y, T y)\}
$$

for some $k \in(0,1)$.

In 1959, Connell [3] gave an example of a metric space $X$ which is not complete and every contraction on $X$ has a fixed point. Also, Subrahmanyam [4] proved the converse of the Banach fixed point theorem using Kannan mapping. Moreover, the assumption of continuity of the mapping and the compactness condition on metric space is required for the existence of a fixed point for a strict type Kannan contraction.

In 2000, Branciari [5] introduced a class of generalised metric spaces by replacing triangular inequality to similar ones which involve four or more points instead of three and improved the Banach contraction mapping principle. This motivated several researchers to prove fixed point results in such spaces. For more details on the fixed point theory of a generalised metric space, we refer to the reader [611]. In 2008, Azam and Arshad [12] using the concept of Branciari [5] investigated fixed points for the mappings given by Kannan [2] by applying the rectangular property in a generalised metric space.

We will require the following definitions and preliminary results to prove our results. 
Definition 2 (see [12]). Let $X$ be a nonempty set. Suppose that the mapping $d: X \times X \longrightarrow R$ satisfies

(i) $d(x, y) \geq 0$, for all $x, y \in X$ and $d(x, y)=0$ if and only if $x=y$

(ii) $d(x, y)=d(y, x)$, for all $x, y \in X$

(iii) $d(x, y) \leq d(x, w)+d(w, z)+d(z, y)$, for all $x, y \in X$ and for all distinct points $w, z \in X-\{x, y\}$ [rectangular property]

Then, $d$ is called a generalised metric and $(X, d)$ is a generalised metric space.

Definition 3 (see [5]). Let $(X, d)$ be a metric space. A mapping $T: X \longrightarrow X$ is said to be sequentially convergent if we have, for every sequence $\left\{y_{n}\right\}$, if $\left\{T y_{n}\right\}$ is convergence then $\left\{y_{n}\right\}$ also is convergence. $T$ is said to be subsequentially convergent if we have, for every sequence $\left\{y_{n}\right\}$, if $\left\{T y_{n}\right\}$ is convergence then $\left\{y_{n}\right\}$ has a convergent subsequence.

Definition 4 (see [13]). Let $X$ be a topological space. If $\left(x_{n}\right)$ is a sequence of points of $X$, and if $n_{1}<n_{2}<\cdots<n_{i}<\cdots$ is an increasing sequence of positive integers, then the sequence $\left(y_{i}\right)$ defined by setting $y_{i}=x_{n_{i}}$ is called a subsequence of the sequence $\left(x_{n}\right)$. The space $X$ is said to be sequentially compact if every sequence of points of $X$ has a convergent subsequence.

For more details on the sequentially convergent property, one can see $[14,15]$.

In 2011, Moradi and Alimohammadi [16] generalised Kannan's results, by using the sequentially convergent mappings and rectangular property in a metric space. Since then, several researchers involved in investigations of Kannan's contraction mappings using a rectangular property in different spaces. For more details, one can see [12, 17-20] and the references therein. Furthermore, Morandi and Alimohammadi [16] investigated and extended Kannan's mapping [2] by using the concept due to Branciari [5]. They proved results on two self-mappings as follows.

Theorem 5 (see [16]). Let $(X, d)$ be a complete metric space and $T, S: X \longrightarrow X$ be mappings such that $T$ is continuous, one-to-one, and subsequentially convergent. If $\lambda \in[0,1 / 2)$ and

$$
d(T S x, T S y) \leq \lambda[d(T x, T S x)+d(T y, T S y)],(x, y \in X),
$$

then $S$ has a unique fixed point. Also, if $T$ is sequentially convergent then for every $x_{0} \in X$, the sequence of iterates $\left\{S^{n} x_{0}\right\}$ converges to this fixed point.

Wardowski [21] gave an interesting generalisation of the Banach fixed point theorem using a different type of contraction called F-contraction. Since then, many researchers following his approach to construct new fixed point theorems for which one can see [22-27] and the references therein.
Wardowski [21] gave the following definitions.

Let $F$ be a function defined as $F: \mathbb{R}^{+} \longrightarrow \mathbb{R}$, which satisfies the following conditions:

(F1) $F$ is strictly increasing, i.e., for all $\alpha, \beta \in \mathbb{R}_{+}$such that $\alpha<\beta, F(\alpha)<F(\beta)$

(F2) For each sequence $\left\{\alpha_{n}\right\}_{n \in \mathbb{N}}$ of positive numbers $\lim _{n \rightarrow \infty} \alpha_{n}=0$ if and only if $\lim _{n \rightarrow \infty} F\left(\alpha_{n}\right)=-\infty$

(F3) There exists $k \in(0,1)$ such that

$$
\lim _{n \rightarrow \infty}\left(\alpha_{n}\right)^{k} F\left(\alpha_{n}\right)=0
$$

Definition 6 (see [21]). A mapping $T: X \longrightarrow X$ is said to be a $F$-contraction if there exists $\tau>0$, such that

$\forall x, y \in X, d(T x, T y)>0 \Longrightarrow \tau+F(d(T x, T y)) \leq F(d(x, y))$.

In 2012, Wardowski [21] introduced a generalization of Banach contraction principle, which is as follows:

Theorem 7 (see [21]). Let $(X, d)$ be a complete metric space and $T: X \longrightarrow X$ be a F-contraction. Then, $T$ has a unique fixed point $x^{*} \in X$ and for every $x_{0} \in X$ a sequence $\left\{T^{n} x_{0}\right\}_{n \in \mathbb{N}}$ is convergent to $x^{*}$.

In 2019, Goswami et al. [22] defined F-contractive type mappings in $b$-metric spaces and proved some fixed point results with suitable examples. Recently, Batra et al. [28] noticed that the definition introduced by Goswami et al. [22] is not meaningful in general. They provided suitable examples to support their opinion on this definition. Therefore, Batra et al. [28] gave the notions of $F$-contraction and Kannan mapping to define a new class of contractions called $F$-Kannan mappings which is in true sense a generalization of Kannan mappings.

Motivated by Batra et al. [28], we use the following notations: Let $X$ be a nonempty set and $(X, d)$ denotes the metric space with metric $d$. Define the cardinality of a set $A$ by card $\{A\}$ and Fix $T$ denotes the set of all fixed points of a mapping $T$.

Batra et al. [28] gave a new generalization family of contraction called F-Kannan mapping and introduced the following definition.

Definition 8 (see [28]). Let $F$ be a mapping satisfying (F1)-(F3). A mapping $T: X \longrightarrow X$ is said to be an F-Kannan mapping if the following holds:

(K1)

$$
T x \neq T y \Longrightarrow T x \neq x \text { or } T y \neq y
$$

(K2) $\exists \Upsilon>0$ such that

$$
\gamma+F(d(T x, T y)) \leq F\left[\frac{d(x, T x)+d(y, T y)}{2}\right]
$$

for all $x, y \in X$, with $T x \neq T y$. 
The remark presented below is due to Batra et al. [28].

Remark 9 (see [28]). By properties of $F$, it follows that every $F$-Kannan mapping $T$ on a metric space $(X, d)$ satisfies the following condition:

$$
d(T x, T y)) \leq \frac{d(x, T x)+d(y, T y)}{2}
$$

for every $x, y \in X$.

Further, it is concluded that $\operatorname{Card}\{\operatorname{Fix} T\} \leq 1$. Let $T$ be a self-map of a metric space $(X, d)$. We say that $T$ is a Picard operator (PO) if $T$ has unique fixed point $x^{*}$ and $\lim _{n \rightarrow \infty} T^{n} x$ $=x^{*}$ for all $x \in X$.

Then, the family of all functions $F: \mathbb{R}^{+} \longrightarrow \mathbb{R}$ satisfying the condition $F_{1}-F_{3}$ is denoted by $\nabla_{F}$.

One can use the following examples in Batra et al. [28] of such functions $F: \mathbb{R}^{+} \longrightarrow \mathbb{R}$ which satisfy $F_{1}-F_{3}$.

Example 10 (see [28]). Let $F_{1}: \mathbb{R}^{+} \longrightarrow \mathbb{R}$ be defined as $F_{1}$ $(z)=\ln (z)$. Then, clearly, (F1)-(F3) are satisfied by $F_{1}(z)$. In fact, (F3) holds for every $k \in(0,1)$,

$$
d(T x, T y) \leq e^{-r}\left[\frac{d(x, T x)+d(y, T y)}{2}\right],
$$

for all $x, y \in X$ with $T x \neq T y$.

Thus, if $T: X \longrightarrow X$ is a Kannan mapping with constant $k \in(0,1)$ satisfying

$$
d(T x, T y) \leq k\left[\frac{d(x, T x)+d(y, T y)}{2}\right],
$$

for every $x, y \in X$.

Example 11 (see [28]). Let $F_{2}: \mathbb{R}^{+} \longrightarrow \mathbb{R}$ be defined as $F_{2}$ $(z)=\ln (z)+z, z>0$. Then, (F1)-(F3) are satisfied by $F_{2}(z)$.

$$
\frac{d(T x, T y)}{(d(x, T x)+d(y, T y)) / 2} e^{d(T x, T y)-[d(x, T x)+d(y, T y)] / 2} \leq e^{-r},
$$

for all $x, y \in X$ with $T x \neq T y$.

The following lemma was proved by Batra et al. [28]

Lemma 12 (see [28]). Let $(X, d)$ be a metric space and $T: X$ $\longrightarrow X$ be an F-Kannan mapping. Then, $d\left(T^{n} x, T^{n+1} x\right) \longrightarrow$ 0 as $n \longmapsto \infty$ for all $x \in X$.

Batra et al. [28] introduced an F-Kannan mapping using the properties by Subrahmanyam [4] which is an extension of Goswami et al. [22] and Wardowski [21] results. They proved the following result.

Theorem 13 (see [28]). Let $(X, d)$ be a complete metric space and suppose $T: X \longrightarrow X$ is an F-Kannan mapping, then $T$ is a Picard operator (PO).
In 2017, Gopal et al. [29] specified the fundamental properties for a fixed point theorem which ensures the existence of a common fixed point for suitable assumptions. Those assumptions are sufficient and include conditions of commutativity, containment of ranges of mappings, continuity of at least one mapping or weaker notion, contractive, and all essential common fixed point theorem attempts to obtain or soften required values of one or more such conditions.

Definition 14 (see [30]). Let $(S, T)$ be a pair of self-mappings on a metric space $(X, d)$. Then, the coincidence point of the pair $(S, T)$ is a point $x \in X$ such that $(S x)=(T x)=x^{*}$, then $x^{*}$ is called coincidence point of the pair $(S, T)$. If $x^{*}=x$, then $x$ is said to be a common fixed point.

Definition 15 (see [31]). Let $S, T$ be self-mappings of a nonempty set $X$. A point $x \in X$ is the coincidence point of $S$ and $T$ if $t=S x=T x$. The set of coincidence point of $S$ and $T$ is denoted by $C(S, T)$.

Definition 16 (see $[31,32])$. Let $(S, T)$ be a pair of selfmappings on a metric space $(X, d)$. Then, the pair $(S, T)$ is said to be

(i) Commuting if, for all $x \in X, S(T x)=T(S x)$,

(ii) Weakly commuting if, for all $d(S(T x), T(S x)) \leq d(S$ $x, T x)$

(iii) Compatible if $\lim _{n \rightarrow \infty} d\left(S T x_{n}, T S x_{n}\right)=0$, whenever $x_{n}$ is a sequence in $X$ such that $\lim _{n \longrightarrow \infty} T x_{n}=$ $\lim _{n \rightarrow \infty} S x_{n}=t$,

(iv) Weakly compatible if, for all $S(T x)=T(S x)$, for every coincidence point $x \in X$.

This paper is aimed at extending and generalising the results due to Batra et al. [28], and Morandi and Alimohammadi [16] using a pair of two self-mappings in F-Kannan mapping. Doing so, we will be able to extend several other results of the same setting in the literature. We will provide some applications of the theorem to the integral equation, nonlinear fractional differential equation, and ordinary differential equation for damped forced oscillations to support the results.

\section{Main Results}

We present the main result of this paper by assuming a map to be sequentially convergent with a pair of two selfmappings in F-Kannan mappings. We shall start with the extension of Definition 8 using a pair of two self-mappings in the F-Kannan mapping setting.

Definition 17. Let $F$ be a mapping satisfying (F1)-(F3). A pair of two self-mapping $T, S: X \longrightarrow X$ is said to be an $F$-Kannan mapping if the following holds: 
$(\mathrm{FK} 1)$

$$
T S x \neq T S y \Longrightarrow T S x \neq x \text { or } T S y \neq y
$$

(FK2) There exists $r>0$ such that

$$
r+F(d(T S x, T S y)) \leq F\left[\frac{d(T x, T S x)+d(T y, T S y)}{2}\right]
$$

for all $x, y \in X$, with $T S x \neq T S y$.

By following Batra et al. [28], we presented the remark as below.

Remark 18. By properties of $F$, it follows that every $F$-Kannan mapping $T$ on a metric space $(X, d)$ satisfies the following condition:

$$
d(T S x, T S y) \leq \frac{d(T x, T S x)+d(T y, T S y)}{2},
$$

for every $x, y \in X$.

We give the following examples in the context of a pair of two mappings:

Example 19. Let $F_{1}: \mathbb{R}^{+} \longrightarrow \mathbb{R}$ be defined as $F_{1}(z)=\ln (z)$. Then, clearly, (F1)-(F3) are satisfied by $F_{1}(z)$. In fact, (F3) holds for every $k \in(0,1)$. Moreover, condition (13) above takes the form:

$$
d(T S x, T S y) \leq e^{-r}\left[\frac{d(T x, T S x)+d(T y, T S y)}{2}\right],
$$

for all $x, y \in X$ with $T S x \neq T S y$.

Thus, if $T, S: X \longrightarrow X$ is a Kannan mapping with constant $k \in(0,1)$ satisfying

$$
d(T S x, T S y) \leq k\left[\frac{d(T x, T S x)+d(T y, T S y)}{2}\right],
$$

for every $x, y \in X$. Then, it also satisfies (15) and (13) with $\gamma=\ln (1 / k)$.

Example 20. Let $F_{2}: \mathbb{R}^{+} \longrightarrow \mathbb{R}$ be defined as $F_{2}(z)=\ln (z)$ $+z, z>0$. Then, (F1)-(F3) are satisfied by $F_{2}(z)$. Condition (13) above takes the form:

$\frac{d(T S x, T S y)}{(d(T x, T S x)+d(T y, T S y)) / 2} e^{d(T S x, T S y)-(d(T x, T S x)+d(T y, T S y)) / 2} \leq e^{-r}$,

for all $x, y \in X$ with $T S x \neq T S y$.

We prove the following lemma which will be useful in proving of the main theorem.
Lemma 21. Let $(X, d)$ be a metric space and $T, S: X \longrightarrow X$ be an F-Kannan mapping. Then,

$$
d\left(T S^{i} x_{0}, T S^{i+1} x_{0}\right) \longrightarrow 0 \text { as } i \longrightarrow \infty
$$

for all $x \in X$.

Proof. Let $x_{0} \in X$ be arbitrary. If $T S^{i} x_{0}=T S^{i+1} x_{0}$ for some $i$ $\in \mathbb{N}$, then sequence $\left\{x_{i}\right\}_{n \in \mathbb{N}}$ converges in $X$ and hence the sequence $d\left(T S^{i} x_{0}, T S^{i+1} x_{0}\right) \longrightarrow 0$ as $i \longrightarrow \infty$ for all $x \in X$.

Assume that $T S^{i} x_{0} \neq T S^{i+1} x_{0}$ for any $i \in \mathbb{N}$. Then, by (13) with $Y>0$, we get

$$
\begin{aligned}
\mathcal{Y} & +F\left(d\left(T S^{i} x_{0}, T S^{i+1} x_{0}\right)\right) \\
& \leq F\left[\frac{d\left(T S^{i-1} x_{0}, T S^{i} x_{0}\right)+d\left(T S^{i} x_{0}, T S^{i+1} x_{0}\right)}{2}\right] .
\end{aligned}
$$

From Remark 18, we have

$d\left(T S^{i} x_{0}, T S^{i+1} x_{0}\right) \leq \frac{d\left(T S^{i-1} x_{0}, T S^{i} x_{0}\right)+d\left(T S^{i} x_{0}, T S^{i+1} x_{0}\right)}{2}$

Using (20) in (19), as results yield to

$\Upsilon+F\left(d\left(T S^{i} x_{0}, T S^{i+1} x_{0}\right)\right) \leq F\left(d\left(T S^{i} x_{0}, T S^{i+1} x_{0}\right)\right)$

Letting $i \longrightarrow \infty$ in $(21)$, we get

$$
\begin{aligned}
\gamma+0 & \leq 0, \\
\gamma & \leq 0,
\end{aligned}
$$

which is a contradiction. Hence, $d\left(T S^{i} x_{0}, T S^{i+1} x_{0}\right) \longrightarrow 0$ as $i \longrightarrow \infty$.

Motivated by Morandi and Alimohammadi [16], we prove the extended version of Theorem 5 using F-Kannan mappings with a pair of two self-mappings in metric space.

Theorem 22. Let $(X, d)$ be a complete metric space and $T, S$ $: X \longrightarrow X$ be an F-Kannan mapping such that $T$ is continuous, injection, and subsequentially convergent. If $\lambda \in[0,1 / 2)$, $r>0$ and

$$
\gamma+F(d(T S x, T S y)) \leq F\left[\frac{d(T x, T S x)+d(T y, T S y)}{2}\right]
$$

then $S$ has a unique fixed point. Also, if $T$ is subsequentially convergent then for every $x_{0} \in X$ the sequence of iterates $\left\{S^{i}\right.$ $\left.x_{0}\right\}$ converges to this fixed point.

Proof. Assume $x_{0} \in X$ be an arbitrary point in $X$. Let the sequence $\left\{x_{i}\right\}_{i \geq 1}$ be defined by $x_{i+1}=S x_{i}$ and $x_{i}=S^{i} x_{0}$, for $i=1,2, \cdots$. 
Using inequality (13), we obtain

$$
\begin{gathered}
d\left(T x_{i}, T x_{i+1}\right)=d\left(T S x_{i-1}, T S x_{i}\right) . \\
r+F\left(d\left(T x_{i}, T x_{i+1}\right)\right) \leq F\left[\frac{d\left(T x_{i-1}, T S x_{i-1}\right)+d\left(T x_{i}, T S x_{i}\right)}{2}\right], \\
F\left(d\left(T x_{i}, T x_{i+1}\right)\right) \leq F\left[\frac{d\left(T x_{i-1}, T S x_{i-1}\right)+d\left(T x_{i}, T S x_{i}\right)}{2}\right]-r .
\end{gathered}
$$

Since $F$ is strictly increasing, by using Remark 9 , we deduce

$$
\begin{aligned}
& d\left(T x_{i}, T x_{i+1}\right)<\frac{d\left(T x_{i-1}, T S x_{i-1}\right)+d\left(T x_{i}, T S x_{i}\right)}{2}, \\
& d\left(T x_{i}, T x_{i+1}\right)<\frac{d\left(T x_{i-1}, T x_{i}\right)+d\left(T x_{i}, T x_{i+1}\right)}{2},
\end{aligned}
$$

and hence

$$
\begin{aligned}
2 d\left(T x_{i}, T x_{i+1}\right)-d\left(T x_{i}, T x_{i+1}\right) & <d\left(T x_{i-1}, T x_{i}\right), \\
d\left(T x_{i}, T x_{i+1}\right) & <d\left(T x_{i-1}, T x_{i}\right) .
\end{aligned}
$$

By (F1), this implies that

$$
F\left(d\left(T x_{i}, T x_{i+1}\right)\right)<F\left(d\left(T x_{i-1}, T x_{i}\right)\right) .
$$

Consequently, we get

$$
\gamma+F\left(d\left(T x_{i}, T x_{i+1}\right)\right) \leq F\left(d\left(T x_{i-1}, T x_{i}\right)\right),
$$

so,

$$
F\left(d\left(T x_{i}, T x_{i+1}\right)\right) \leq F\left[d\left(T x_{i-1}, T x_{i}\right)\right]-r .
$$

Similarly, we obtain

$$
\begin{aligned}
d\left(T x_{i+1}, T x_{i+2}\right) & =d\left(T S x_{i}, T S x_{i+1}\right), \\
F\left(d\left(T x_{i+1}, T x_{i+2}\right)\right. & \leq F\left[\frac{d\left(T x_{i}, T S x_{i}\right)+d\left(T x_{i+1}, T S x_{i+1}\right)}{2}\right]-r,
\end{aligned}
$$

which implies that

$$
F\left(d\left(T x_{i+1}, T x_{i+2}\right)\right) \leq F\left[d\left(T x_{i}, T x_{i+1}\right)\right]-r .
$$

Applying (29) in (31), we obtain

$$
F\left(d\left(T x_{i+1}, T x_{i+2}\right)\right) \leq F\left[d\left(T x_{i-1}, T x_{i}\right)\right]-2 \Upsilon,
$$

by (F1).

Using induction and (29), we deduce

$$
F\left(d\left(T x_{i}, T x_{i+1}\right)\right) \leq F\left[d\left(T x_{i-1}, T x_{i}\right)\right]-i \Upsilon .
$$

Letting $i \longrightarrow \infty$ in (33) and using property (F2) of $F$ results in

$$
\lim _{n \rightarrow \infty} d\left(T x_{i}, T x_{i+1}\right)=0
$$

By Lemma 21, we have $d\left(T x_{i}, T x_{i+1}\right) \longrightarrow 0$ as $i \longrightarrow \infty$. Denote $d\left(T x_{i}, T x_{i+1}\right)=\alpha_{i}$, for all $i=1,2,3, \cdots$ and $i \in \mathbb{N}$, for $F$-Kannan mappings.

Using condition $\left(F_{3}\right)$ of the function $F$ there exists $k \in($ $0,1)$ such that

$$
\lim _{n \rightarrow \infty}\left(\alpha_{i}\right)^{k} F\left(\alpha_{i}\right)=0
$$

From (33), for every $i \in \mathbb{N}$, we have

$$
\begin{aligned}
& \left(\alpha_{i}\right)^{k} F\left(\alpha_{i}\right) \leq \cdots \leq\left(\alpha_{n}\right)^{k} F\left(\alpha_{i-1}\right)-i \Upsilon\left(\alpha_{i}\right)^{k}, \\
& \left(\alpha_{i}\right)^{k} F\left(\alpha_{i}\right)-\left(\alpha_{i}\right)^{k} F\left(\alpha_{i-1}\right) \leq-i \Upsilon\left(\alpha_{i}\right)^{k}, \\
& \left(\alpha_{i}\right)^{k}\left[F\left(\alpha_{i}\right)-F\left(\alpha_{i-1}\right)\right] \leq-i \Upsilon\left(\alpha_{i}\right)^{k} \leq 0 .
\end{aligned}
$$

On taking limit as $i \longrightarrow \infty$ in (36), we get

$$
\lim _{i \rightarrow \infty} i\left(\alpha_{i}\right)^{k}=0 .
$$

From (39), there exist $i_{1} \in \mathbb{N}$ such that $i\left(\alpha_{i}\right)^{k} \leq 1$, for all $i \geq i_{1}$, which follows that

$$
\alpha_{i} \leq i^{-1 / k}, \forall i \geq i_{1} .
$$

Therefore, $\sum_{i=0}^{\infty} d\left(T x_{i}, T x_{i+1}\right)$ converges.

By (40), we prove that $\left\{T x_{i}\right\}$ is a Cauchy sequence since $(X, d)$ is complete. Consider $i, j \in N$ such that $j \geq i$,

$$
\begin{aligned}
d\left(T x_{i}, T x_{j}\right) \leq & d\left(T x_{i}, T x_{i+1}\right)+d\left(T x_{i+1}, T x_{i+2}\right) \\
& +d\left(T x_{i+2}, T x_{i+3}\right)+\cdots+d\left(T x_{j-1}, T x_{j}\right), \\
\leq & \alpha_{i}+\alpha_{i+1}+\alpha_{i+2}+\cdots+\alpha_{j-1}, \\
= & \sum_{i}^{j-1} d\left(T x_{i}, T x_{i+1}\right) \leq \sum_{j=i}^{\infty} \alpha_{i} \leq \sum_{i=1}^{\infty} i^{-1 / k} .
\end{aligned}
$$

This shows that the series $\sum_{i=1}^{\infty} i^{-1 / k}$ converges, which implies that

$$
\lim _{i \rightarrow \infty} d\left(T x_{i}, T x_{j}\right)=0 .
$$

So, $T x_{i}=T x_{j}$ for every $j \geq i$ in $X$. Hence, $\left\{T x_{i}\right\}$ is a Cauchy sequence in $X$. The completeness of $X$ ensures the existence of $x^{*} \in X$ such that

$$
d\left(T x^{*}, x^{*}\right)=\lim _{i, j \rightarrow \infty} d\left(T x_{i}, T x_{j}\right)=0,=\lim _{i \rightarrow \infty} d\left(T x_{i}, x^{*}\right)=0 .
$$


By (43), it follows that $x_{i+1} \longrightarrow x^{*}$ as $i \longrightarrow \infty$. By continuity of $S$ and $T$, we have

$$
\begin{aligned}
& x^{*}=\lim _{i \rightarrow \infty} x_{i}=\lim _{i \rightarrow \infty} x_{i+1}=\lim _{i \rightarrow \infty} S x_{i}=S x^{*}, \\
& x^{*}=\lim _{i \rightarrow \infty} x_{i}=\lim _{i \rightarrow \infty} x_{i+1}=\lim _{i \rightarrow \infty} T x_{i}=T x^{*} .
\end{aligned}
$$

Since $X$ is a complete metric space, there exists $x^{*} \in X$ such that

$$
\lim _{i \rightarrow \infty} T x_{i}=x^{*}
$$

Now, we prove that $x^{*}$ is a fixed point of $T$. Thus, by (iii) of Definition 2, we have

$$
d\left(x^{*}, T x^{*}\right) \leq d\left(x^{*}, T x_{i}\right)+d\left(T x_{i}, T x_{i+1}\right)+d\left(T x_{i+1}, T x^{*}\right) .
$$

By Remark 18, it implies that

$$
d\left(T x_{i+1}, T x^{*}\right) \leq \frac{d\left(T x_{i}, T x_{i+1}\right)+d\left(T x_{i+1}, T x^{*}\right)}{2} .
$$

Applying (47) in (46), we obtain

$$
\begin{aligned}
d\left(x^{*}, T x^{*}\right) \leq & d\left(x^{*}, x^{*}\right)+d\left(x^{*}, x^{*}\right) \\
& +\frac{d\left(x^{*}, T x^{*}\right)+d\left(x^{*}, T x^{*}\right)}{2} .
\end{aligned}
$$

Letting $i \longrightarrow \infty$ and using Lemma 21 in above inequality, we get

$$
\begin{aligned}
d\left(x^{*}, T x^{*}\right) \leq & d\left(x^{*}, T x_{i}\right)+d\left(T x_{i}, T x_{i+1}\right) \\
& +\frac{d\left(T x_{i}, T x_{i+1}\right)+d\left(T x_{i+1}, T x^{*}\right)}{2}, \\
\leq & d\left(x^{*}, x^{*}\right)+d\left(x^{*}, x^{*}\right) \\
& +\frac{d\left(x^{*}, x^{*}\right)+d\left(x^{*}, T x^{*}\right)}{2}, \\
d\left(x^{*}, T x^{*}\right) \leq & \frac{d\left(x^{*}, T x^{*}\right)}{2}, \\
2 d\left(x^{*}, T x^{*}\right) \leq & d\left(x^{*}, T x^{*}\right), \\
2 d\left(x^{*}, T x^{*}\right) \leq & d\left(x^{*}, T x^{*}\right), \\
d\left(x^{*}, T x^{*}\right) \leq & 0 .
\end{aligned}
$$

That is, $T x^{*}=x^{*}$.

Next, we prove that $x^{*}$ is a unique fixed point of $T$. Assume the contrary, i.e, there exists $w^{*} \in \operatorname{Card}\{$ Fix $T\}$ such that $x^{*} \neq w^{*}$. Let $T x_{i} \longrightarrow w^{*}$ and $w^{*}$ is a fixed point of $T$. Using Remark 18 and Lemma 21, it follows that $x^{*}=w^{*}$ which is a contradiction. Thus, $T$ is a PO on $X$.

Moreover, $T$ is a subsequentially convergent, $\left\{x_{i}\right\}$ has a convergent subsequence, and there exists $w^{*} \in X$ and $\left\{x_{i(k)}\right\}_{k=1}^{\infty}$ so that $\lim _{k \rightarrow \infty} x_{i(k)}=w^{*}$. Since $T$ is continuous and

$$
\lim _{k \rightarrow \infty} x_{i(k)}=w^{*}
$$

Due to the continuity of $T$, it implies that

$$
\lim _{k \rightarrow \infty} T x_{i(k)}=T w^{*}
$$

By (45), we conclude that

$$
T w^{*}=x^{*}
$$

Using Remark 18 and (ii) of Definition 2, we get

$$
\begin{aligned}
d\left(T x_{i}, T x_{i+1}\right) & =d\left(T S x_{i-1}, T S x_{i}\right) \\
& \leq \lambda\left(d\left(T x_{i-1}, T S x_{i-1}\right)+d\left(T x_{i}, T S x_{i}\right)\right), \\
& =\lambda\left(d\left(T x_{i-1}, T S x_{i-1}\right)+d\left(T x_{i}, T x_{i+1}\right)\right) \\
& \leq \frac{\lambda}{1-\lambda} d\left(T x_{i-1}, T S x_{i-1}\right) .
\end{aligned}
$$

Thus, using equation (13) and (iii) of Definition 2, we have

$$
\begin{gathered}
\Upsilon+F\left(d\left(T x_{i+1}, T x_{i+2}\right)\right)=\gamma+F\left(d\left(T S x_{i}, T S x_{i+1}\right)\right) \\
F\left(d\left(T S w^{*}, T w^{*}\right)\right) \leq \\
+F\left(d\left(T S w^{*}, T x_{i}\right)+d\left(T x_{i}, T x_{i+1}\right)\right. \\
\left.\left.+d\left(T x_{i+1}\right), T w^{*}\right)\right)
\end{gathered}
$$

As $F$ is sequentially increasing, this implies that

$$
\begin{aligned}
d\left(T S w^{*}, T w^{*}\right)< & d\left(T S w^{*}, T x_{i}\right)+d\left(T x_{i}, T x_{i+1}\right) \\
& +d\left(T x_{i+1}, T w^{*}\right), d\left(T S w^{*}, T w^{*}\right) \\
\leq & d\left(T S w^{*}, T S^{i(k)} x_{0}\right) \\
& +d\left(T S^{i(k)} x_{0}, T S^{i(k)+1} x_{0}\right) \\
& +d\left(T S^{i(k)+1} x_{0}, T w^{*}\right) .
\end{aligned}
$$

By Lemma 21 when $T S^{i} x_{0} \neq T S^{i+1} x_{0}$ for any $i \in \mathbb{N}$ and (13), we obtain

$$
\begin{aligned}
d\left(T S w^{*}, T S^{i(k)} x_{0}\right) \leq & \lambda\left[d\left(T w^{*}, T S w^{*}\right)\right. \\
& \left.+d\left(T S^{i(k)-1} x_{0}, T S^{i(k)} x_{0}\right)\right] \\
\leq & \lambda d\left(T w^{*}, T S w^{*}\right) \\
& +\lambda\left(\frac{\lambda}{1-\lambda}\right)^{i(k)-1} d\left(T x_{0}, T x_{1}\right),
\end{aligned}
$$

$d\left(T S^{i(k)+1} x_{0}, T S^{i(k)} x_{0}\right) \leq\left(\frac{\lambda}{1-\lambda}\right)^{i(k)} d\left(T x_{1}, T x_{0}\right)$ 
Using (56) and (57) in (55), we obtain

$$
\begin{aligned}
& d\left(T S w^{*}, T w^{*}\right) \\
& \leq \lambda d\left(T w^{*}, T S w^{*}\right)+\lambda\left(\frac{\lambda}{1-\lambda}\right)^{i(k)-1} d\left(T x_{0}, T x_{1}\right) \\
& \quad+\left(\frac{\lambda}{1-\lambda}\right)^{i(k)} d\left(T x_{1}, T x_{0}\right)+d\left(T x_{i(k)+1}, T w^{*}\right)
\end{aligned}
$$

which follows

$$
\begin{aligned}
& d\left(T S w^{*}, T w^{*}\right) \\
& \leq\left(\frac{\lambda}{1-\lambda}\right)^{i(k)} d\left(T x_{0}, T x_{1}\right)+\left(\frac{\lambda}{1-\lambda}\right)^{i(k)+1} d\left(T x_{1}, T x_{0}\right) \\
& \quad+\left(\frac{1}{1-\lambda}\right) d\left(T x_{i(k)+1}, T w^{*}\right) .
\end{aligned}
$$

Letting $k \longrightarrow \infty$ in (59), we obtain

$$
d\left(T S w^{*}, T w^{*}\right)=0
$$

Since $T$ is injection, $S w^{*}=w^{*}$. So, $S$ has a fixed point. As $T$ is sequentially convergent, we conclude that $\left\{x_{i}\right\}$ converges to the fixed point of $S$. Implying that $S x \subset X$ and $T x$ $\subset X$, then, there exists a point $w^{*} \subset X$ such that $w^{*} \in S w^{*} \cap$ $T w^{*}$, that is, $w^{*}$ is a common fixed point of $S$ and $T$. which satisfies all fundamental property of Definition 16.

Dasgupta et al. [33] and Moradi and Alimohammadi [16], in their work, considered an example in which Kannan Theorem is not applicable. At the same time, generalised Kannan mappings imply the existence of a fixed point for the considered mapping. In this work, we use one more example of this type which satisfies F-Kannan mapping.

Example 23. Consider the sequence $X=\{0\} \cup\{1,1 / 2,1 / 3$, $\cdots\}$ and $d$ be a Euclidean metric on $X$. Then, $(X, d)$ is a complete metric space. Let the mapping $S: X \longrightarrow X$ be determined as follows:

$$
\begin{aligned}
S(0) & =0, \\
S\left(\frac{1}{n}\right) & =\frac{1}{n+1} .
\end{aligned}
$$

for $n \geq 1$. Let there exists $\lambda \in[0,1 / 2)$, so that for all $x, y \in X$ condition (1) is satisfied although is not true for every $\lambda>0$ , which is a contradiction, hence, Kannan's theorem cannot be applicable.

The mapping $T: X \longrightarrow X$ be determined as

$$
\begin{gathered}
T(0)=0, \\
T\left(\frac{1}{n}\right)=\frac{1}{n^{n}} .
\end{gathered}
$$

For all $n>1, T$ is continuous, injection, and subsequentially convergent.

Now, let $m, n \in \mathbb{N}, m>n$. Then, we prove that $(T, S)$ is an $F$-Kannan mapping with respect to $F_{2}(z)=\ln z+z$ and $Y$ $=1$.

By using (KF2) with $F_{2}(z)$, we note that (23) becomes

$\frac{d(T S x, T S y)}{[d(T x, T S x)+d(T y, T S y)] / 2} e^{d(T S x, T S y)-[(d(T x, T S x)+d(T y, T S y)) / 2]} \leq e^{-r}$

To see this, we now calculate $d(S x, T y)$ for $x=1 / m, y=$ $1 / n, n \geq 1$.

$$
\begin{aligned}
d(T S x, T S y) & =d\left(T S\left(\frac{1}{m}\right), T S\left(\frac{1}{n}\right)\right), \\
& \leq\left|\frac{1}{(n+1)^{n+1}}-\frac{1}{(m+1)^{m+1}}\right|, \\
d(T x, T S x) & =d\left(T\left(\frac{1}{m}\right), T S\left(\frac{1}{m}\right)\right), \\
& \leq \frac{1}{3}\left|\frac{1}{m}-\frac{1}{(m+1)^{m+1}}\right|, \\
d(T y, T S y) & =d\left(T\left(\frac{1}{n}\right), T S\left(\frac{1}{n}\right)\right), \\
& \leq \frac{1}{3}\left|\frac{1}{n}-\frac{1}{(n+1)^{n+1}}\right| .
\end{aligned}
$$

Applying (64),(65), and (66) in (63) becomes

$$
\begin{aligned}
& \frac{|T S(1 / m), T S(1 / n)|}{[|T(1 / m), T S(1 / m)|+|T(1 / n), T S(1 / n)|] / 6} \\
& \cdot e^{|T S(1 / m), T S(1 / n)|-[|T(1 / m), T S(1 / m)|+|T(1 / n), T S(1 / n)| / 6]} \leq e^{-r} .
\end{aligned}
$$

By Theorem 22, S has unique fixed point that is $x^{*}=0$.

\section{Some Applications}

In this section, we will provide three applications of the theorem proved in the previous section.

3.1. Existence of a Solution for an Integral Equation. In this section, as inspired by Nashine et al. [34], we establish the existence of a solution for the following Volterra type integral equation: This problem is equivalent to the integral equation

$$
u(t)=h(t)+\int_{a}^{b} f(t, s, u(s)) d s, \forall t, s \in[a, b],
$$

where $f:[a, b] \times[a, b] \times \mathbb{R} \longrightarrow \mathbb{R}$ and $h:[a, b] \longrightarrow \mathbb{R}$ are continuous functions. Let $X=C([a, b], \mathbb{R})$ be the space of all continuous functions defined on $C[a, b]$. Notice that $C[a, b]$ endowed with metric 


$$
d(x, y)=\|x-y\|_{\infty}=\widehat{\mathrm{A}} \max _{t \in[a, b]}|x(t)-\widehat{\mathrm{A}} y(t)|,
$$

is a complete metric space and $X$ can be equipped with the partial order $\preccurlyeq$ given by $x, y \in X,(x \preccurlyeq y) \Longrightarrow(x(t) \preccurlyeq y(t$ ) and $\left.\|x\|_{\infty},\|y\|_{\infty} \leq 1\right)$, or $(x(t)=y(t))$ for all $t \in[a, b]$. It was shown by Nieto and Rodrigurz-Lopez [35] that $(X$, $\preccurlyeq)$ is regular. For more applications to nonlinear integral equations, one can see [36-38] and the references therein.

Now, we define a mapping $S: X \longrightarrow X$ by

$$
S x(t)=h(t)+\int_{a}^{t} f(t, s, x(s)) d s, t \in[a, b] .
$$

If $x \in[a, b]$ is a fixed point of $S$, then $x \in[a, b]$ is a solution of (68).

We prove our results, by establishing the existence of a common fixed point for a pair of self-mappings.

Theorem 24. Let $S, T: C([a, b]) \longrightarrow C([a, b])$ be self-maps of a metric space $(X, d)$ such that the following condition holds

(1) For all $t, s \in[a, b]$ and $u \in C[a, b]$, we have

$$
f(t, s, u(t)) \leq f\left(t, s \int_{a}^{b} f(s, \tau, u(\tau)) d \tau+h(t)\right)
$$

(2) There exists two functions $f_{1}, f_{2}:[a, b] \times X \times X \longrightarrow X$ with constants $\alpha$ such that, for all $t \in[a, b]$, we have

$$
\left|f_{1}(t, s, a)\right|-\left|f_{2}(t, s, b)\right| \leq \alpha(t, s) q|b-a|, q \leq 1
$$

(3) For

$$
\sup _{t \in[a, b]} \int_{a}^{b} \alpha(t, s) d s \leq 1
$$
$, \mathbb{R})$.

Then, integral equation (68) has a solution $x^{*} \in C([a, b]$

Proof. From (a), for all $t \in[a, b]$, we have

$$
\begin{aligned}
S x(t) & =h(t)+\int_{a}^{b} f(t, s, x(s)) d s, \forall t, s \in[a, b] \\
& \leq f\left(t, s \int_{a}^{b} f(s, \tau, x(\tau)) d \tau+h(s)\right) d s+h(t) \\
& =h(t)+\int_{a}^{b} f(t, s, S x(s)) d s, \forall t, s \in[a, b] \\
& =S(S x)(t) .
\end{aligned}
$$

Therefore, $S x \leq S(S x)$ for all $x \in[a, b]$.
By using condition (2) of Theorem 24, we obtain

$$
\begin{aligned}
|S x(t)-S y(t)| & =\int_{a}^{b}|f(t, s, x(s))-f(t, s, y(s))| d s, \\
& \leq \int_{a}^{b}|\alpha(t, s) q| d s(\alpha|x(t)-y(t)|), \\
& =q d(x, y) \int_{a}^{b}|\alpha(t, s)| d s, \leq q d(x, y) .
\end{aligned}
$$

Thus, $d(S x, S y) \leq q d(x, y)$ for all $x, y \in X$. Hence, $x=x^{*}$ is a common fixed point of $S$ and $T$, also a solution to integral equation. Then, the integral equation (68) has a solution $x^{*}$ $\in C([a, b], \mathbb{R})$.

3.2. Existence of a Solution for Nonlinear Fractional Differential Equation. The purpose of this section is to provide an application of Theorem 22 to get a common solution of a nonlinear fractional differential equation, where we can apply $F$-Kannan mappings in metric spaces.

Here, we investigates the Caputo derivative with the fractional order of the nonlinear fractional differential equation. This form of fractional derivative for a continuous function $f:[0, \infty) \longrightarrow \mathbb{R}$ is given as

$$
\begin{gathered}
\left({ }^{C} D_{t}^{\alpha}\right) f(t)=\frac{1}{\Gamma(n-\alpha)} \int_{a}^{t}(t-s)^{n-\alpha-1} f^{n}(s) d s, \\
\quad(n-1<\alpha, n=[\alpha]+1),
\end{gathered}
$$

where $[\alpha]$ denotes the integer part of the real number $\alpha$ (see $[37,39])$. Also, the Riemann-Liouville fractional integral of order $\alpha$ is given by

$$
\left(I_{s}^{\alpha}\right) f(t)=\frac{1}{\Gamma(\alpha)-1} \int_{0}^{t}(t-s)^{\alpha} f(s) d s(\alpha>0) .
$$

The Caputo fractional differential equation has several applications in mathematics, i.e., in image processing, digital data processing, electrical signal, acoustics, physics, and probability theory (one can see in [40]). The following nonlinear fractional differential equation is inspired by Kilbas et al. [41], Baleanu et al. [39], Budhia et al. [42], and Kanwal et al. [37]:

$$
\left\{\begin{array}{l}
{ }^{C} D_{s}^{\alpha} x(t)=f(t, x(t)), t \in(0,1), 1<\alpha \leq 2, \\
x(0)=0, x(1)=\int_{0}^{v} x(s) d s(0<v<1),
\end{array}\right.
$$

where ${ }^{C} D_{s}^{\alpha}$ denotes the Caputo fractional derivative of order $\alpha$ and $f:[0,1] \longrightarrow X$ is a continuous function.

Consider the space $X=C(I)(I=[0,1])$ of the continuous function defined on $I$. Suppose that $(X, \|)$ is a Banach space, and $I:=[0, T], T>0$. Let $C(I, X)$ be the Banach space of all continuous functions from $I$ into $X$ with the norm $\|x\|:=$ sup $|x(t)|=L, t \in I$ for $x \in C(I, X)$ (one can see in [43]). 
This space defines the metric by

$$
d(x, y)=\sup _{t \in[0, T]}\{|x(t)-y(t)|\}
$$

$\forall x, y \in X$. This is a complete metric space.

Nonlinear fractional equation (78) can be written as

$$
\begin{aligned}
x(t)= & \frac{1}{\Gamma(\alpha)} \int_{0}^{t}(t-s)^{\alpha-1} f(s, x(s)) d s \\
& -\frac{2 t}{\left(2-v^{2}\right) \Gamma(\alpha)} \int_{0}^{1}(1-s)^{\alpha-1} f(s, x(s)) d s \\
& +\frac{2 t}{\left(2-v^{2}\right) \Gamma(\alpha)} \int_{0}^{v}\left[\int_{0}^{s}(s-z)^{\alpha-1} f(z, x(z)) d z\right] d s .
\end{aligned}
$$

A function $x \in C(I, X)$ is a solution of the fractional differential integral equation (80) if and only if $x$ is a solution of the nonlinear fractional differential equation (78).

Now, we prove the following theorem.

Theorem 25. Suppose the following condition hold:

(i) $f \in C(I \times X, X)$ is sequentially continuous

(ii) There exists a continuous function $f:[0,1] \times \mathbb{R} \longrightarrow$ $\mathbb{R}_{+}$, such that

$$
\mid f\left(t, x(s)-f\left(t, y(s)\left|\leq e^{-\gamma} L\right| x(s)-y(s) \mid\right.\right.
$$

for all $t \in[0,1]$ and for all $x, y \in X$ such that $d(x(t), y$ $(t)) \geq 0$ and a constant $L$ with a constant $\kappa \in L$, such that

$$
\begin{gathered}
L \kappa \leq 1 \\
\kappa=\frac{t^{\alpha}\left(2-v^{2}\right)(\alpha+1)+2 t\left(\alpha+v^{\alpha+1}+1\right)}{\left(2-v^{2}\right) \Gamma(\alpha) \alpha(\alpha+1)}
\end{gathered}
$$

Then, fractional differential equation (78) has a common solution as a fixed point $x^{*} \in C(I, X)$.

Proof. Let us define $T, S: C(I) \longrightarrow C(I)$ by

$$
\begin{aligned}
T S x(t)= & \frac{1}{\Gamma(\alpha)} \int_{0}^{t}(t-s)^{\alpha-1} f(s, x(s)) d s \\
& -\frac{2 t}{\left(2-v^{2}\right) \Gamma(\alpha)} \int_{0}^{1}(1-s)^{\alpha-1} f(s, x(s)) d s \\
& +\frac{2 t}{\left(2-v^{2}\right) \Gamma(\alpha)} \int_{0}^{v}\left[\int_{0}^{s}(s-z)^{\alpha-1} f(z, x(z)) d z\right] d s
\end{aligned}
$$

for $t \in[0,1]$, then TS is sequentially continuous. Suppose that

$$
S x(t)=\int_{0}^{s}(s-z)^{\alpha-1} f(z, x(z)) d z,
$$

this implies that $S \in T S$ and $S$ posses a fixed point $x^{*} \in T S$. To prove the existence of fixed point of TS, we prove that TS is sequentially and contraction. To show TS is sequentially continuous, let $T S x \neq T S y$, for all $x, y \in[0, T]$. By condition (ii), we have

$$
\begin{aligned}
& |T S x-T S y|=\mid \frac{1}{\Gamma(\alpha)} \int_{0}^{t}(t-s)^{\alpha-1} f(s, x(s)) d s \\
& -\frac{2 t}{\left(2-v^{2}\right) \Gamma(\alpha)} \int_{0}^{1}(1-s)^{\alpha-1} f(s, x(s)) d s \\
& +\frac{2 t}{\left(2-v^{2}\right) \Gamma(\alpha)} \int_{0}^{v}\left[\int_{0}^{s}(s-z)^{\alpha-1} f(z, x(z)) d z\right] d s \\
& -\frac{1}{\Gamma(\alpha)} \int_{0}^{t}(t-s)^{\alpha-1} f(s, y(s)) d s \\
& +\frac{2 t}{\left(2-v^{2}\right) \Gamma(\alpha)} \int_{0}^{1}(1-s)^{\alpha-1} f(s, y(s)) d s \\
& -\frac{2 t}{\left(2-v^{2}\right) \Gamma(\alpha)} \int_{0}^{v}\left[\int_{0}^{s}(s-z)^{\alpha-1} f(z, y(z)) d z\right] d s \mid \text {, } \\
& \leq \frac{1}{\Gamma(\alpha)} \int_{0}^{t}(t-s)^{\alpha-1}|f(s, x(s))-f(s, y(s))| d s \\
& +\frac{2 t}{\left(2-v^{2}\right) \Gamma(\alpha)} \int_{0}^{1}(1-s)^{\alpha-1}|f(s, x(s))-f(s, y(s))| d s, \\
& +\frac{2 t}{\left(2-v^{2}\right) \Gamma(\alpha)} \int_{0}^{v}\left[\int_{0}^{s}(s-z)^{\alpha-1} \mid f(z, x(z))\right. \\
& -f(z, y(z)) \mid d z] d s, \leq \frac{1}{\Gamma(\alpha)} \int_{0}^{t}(t-s)^{\alpha-1}|x(s)-y(s)| d s \\
& +\frac{2 t}{\left(2-v^{2}\right) \Gamma(\alpha)} \int_{0}^{1}(1-s)^{\alpha-1}|x(s)-y(s)| d s \\
& +\frac{2 t}{\left(2-v^{2}\right) \Gamma(\alpha)} \int_{0}^{v}\left[\int_{0}^{s}(s-z)^{\alpha-1}|x(z)-y(z)| d z\right] d s, \\
& =\frac{e^{-r} L}{\Gamma(\alpha)}\|x-y\|_{\infty} \int_{0}^{t}(t-s)^{\alpha-1} d s \\
& +\frac{2 t e^{-\gamma} L}{\left(2-v^{2}\right) \Gamma(\alpha)}\|x-y\|_{\infty} \int_{0}^{1}(1-s)^{\alpha-1} d s \\
& +\frac{2 t e^{-r} L}{\left(2-v^{2}\right) \Gamma(\alpha)}\|x-y\|_{\infty} \int_{0}^{v}\left[\int_{0}^{s}(s-z)^{\alpha-1} d z\right] d s, \\
& \leq\left[\frac{e^{-r} L t^{\alpha}}{\alpha \Gamma(\alpha)}+\frac{2 t e^{-r} L}{\left(2-v^{2}\right) \alpha \Gamma(\alpha)}\right. \\
& \left.+\frac{2 t e^{-r} L v^{\alpha+1}}{\left(2-v^{2}\right) \alpha(\alpha+1) \Gamma(\alpha)}\right]\|x-y\|_{\infty}, \\
& \leq e^{-\gamma} L\left[\frac{t^{\alpha}}{\alpha \Gamma(\alpha)}+\frac{2 t}{\left(2-v^{2}\right) \alpha \Gamma(\alpha)}\right. \\
& \left.+\frac{2 t v^{\alpha+1}}{\left(2-v^{2}\right) \alpha(\alpha+1) \Gamma(\alpha)}\right]\|x-y\|_{\infty}, \\
& =e^{-r} L\left[\frac{t^{\alpha}\left(2-v^{2}\right)(\alpha+1)+2 t\left(\alpha+v^{\alpha+1}+1\right)}{\left(2-v^{2}\right) \Gamma(\alpha) \alpha(\alpha+1)}\right]\|x-y\|_{\infty} \text {, } \\
& \leq e^{-r} L \kappa\|x-y\|_{\infty} \text {. }
\end{aligned}
$$


This implies that

$$
\|T S x, T S y\|_{\infty} \leq e^{-r} L \kappa\|x-y\|_{\infty} .
$$

Since $L \kappa<1$, we have

$$
\|T S x, T S y\|_{\infty} \leq e^{-r}\|x-y\|_{\infty} .
$$

Thus, for each $x, y \in X$, we have

$$
d(T S x, T S y) \leq e^{-r} \mathbb{M}(x, y) .
$$

Taking logarithms on both sides of (88) using $F_{1}(z)=$ $\ln (z)$ and the property of $F$, we get

$$
\ln (d(T S x, T S y)) \leq \ln \left(e^{-r} \mathbb{M}(x, y)\right) .
$$

Equivalently to

$$
r+F(d(T S x, T S y)) \leq F(\mathbb{M}(x, y)) .
$$

For $L \kappa \in[0,1), \gamma>0$, and $\mathbb{M}(x, y)$ is an $F$-Kannan mapping. Therefore, TS is a contraction mapping on $X$. Since all the conditions of Theorem 25 are satisfied, therefore, there exists $x^{*} \in C(I)$ a common fixed point of $T$ and $S$, that is, $x^{*}$ is a solution to fractional nonlinear differential equation (78).

3.3. Existence of Common Solution of Ordinary Differential Equations for Damped Forced Oscillations. This section investigates the solution of the forced damped oscillation differential equations problem in the setting of metric spaces. Nieto and Rodríguez-López [35] initiated the proof of the existing solution of an ordinary differential equation. Since then, several authors are interested in this line of research. In details, one can see the literature in $[41,44-48]$ and the references therein.

In 2020, Shoib et al. [49], considered the forced damped oscillation differential problem of an object of mass $m$ moves to and fro on the $x$-axis around an equilibrium position $x=0$. The object has position $x(t)$ at time $t$, it undergoes the applied force $f(t)$ such that

$$
m \frac{d^{2} x}{d t^{2}}+b \frac{d x}{d t}+k x=f(t) .
$$

where $m, b$, and $k$ are constant positive numbers. If the intial conditions are assumed to be

$$
x(0)=0, x^{\prime}(0)=0 .
$$

Problem (91) can be written as

$$
x(t)=\int_{0}^{T} G(t, s) K(t, x(s)) d s, \forall t, s \in[0, T],
$$

where $T>0$. Let $X=C(I), I=[0, T]$.
The green function for forced damped oscillation is defined by

$$
G(t, s)= \begin{cases}-s e^{\tau(T+s-t)}, & 0 \leq s \leq t \leq T, \\ -t e^{\tau(t-s)}, & 0 \leq t \leq s \leq T,\end{cases}
$$

where $\tau$ can be written in terms of $b, k$, and $m$.

Inspired by Shoaib et al. [49] and Gupta et al. [45], we find the common solution of a forced damped oscillation differential equation using the fixed point method.

Theorem 26. Suppose the following assumption hold:

(i) There exists a continuous function $K:[0, T]^{2} \longrightarrow[0$, $\infty)$, such that

$$
|K(t, s, x(t))-K(t, s, y(t))| \leq e^{-r} \sqrt{\frac{\ln \left[(x-y)^{2}+1\right]}{x-y}},
$$

for $t, s \in[0, T], r>0$ and $x, y \in \mathbb{R}$, where,

$$
\sqrt{\frac{\ln \left[(x-y)^{2}+1\right]}{x-y}}=\frac{d(T x, T S x)+d(T y, T S y)}{2}
$$

(ii) There exist a functon $G: I \times I \longrightarrow \mathbb{R}_{+}$, such that

$$
\sup _{t \in[0, T]} \int_{0}^{T} G^{2}(t, s) d s \leq \frac{1}{T}
$$

Then, problem (93) has a fixed point $x^{*} \in X$, which is a solution of (91).

Proof. Let TS: $C^{1}([0,1]) \longrightarrow C^{1}([0,1])$ be an operator defined by

$$
T S x(t)=\int_{0}^{T} G(t, s) K(t, s, x(s)) d s .
$$

Consider $x>y$, for all $x, y \in C(I)$ and (93), we have

$$
\begin{aligned}
& |T S x(t)-T S y(t)| \\
& \quad=\sup _{t \in[0,1]} \int_{0}^{T} G(t, s)[K(t, s, x(s))-K(t, s, y(s))] d s, \\
& \quad \leq \sup _{t \in[0, T]} \int_{0}^{t} e^{-r} G(t, s) \sqrt{\frac{\ln \left[(x(s)-y(s))^{2}+1\right]}{x(s)-y(s)}} d s .
\end{aligned}
$$

Recall Cauchy-Schwartz inequality defined by

$$
\left(\sum_{i=1}^{\infty}\left|x_{i} y_{i}\right|\right)^{2} \leq\left(\sum_{i=1}^{\infty}\left|x_{i}\right|^{2}\right)\left(\sum_{i=1}^{\infty}\left|y_{i}\right|^{2}\right) .
$$


Using (99) in (100), we obtain

$$
\begin{aligned}
& \left(\int_{0}^{T} e^{-r} G(t, s) \sqrt{\frac{\ln \left[(x(s)-y(s))^{2}+1\right]}{x(s)-y(s)}}\right) \\
& \quad \leq\left(\int_{0}^{T} G^{2}(t, s)\right)^{1 / 2}\left(\int_{0}^{T}\left[e^{-2 r} \sqrt{\frac{\ln \left[(x(s)-y(s))^{2}+1\right]}{x(s)-y(s)}}\right]^{2}\right)^{1 / 2} .
\end{aligned}
$$

Then,

$$
\int_{0}^{T} G^{2}(t, s)=\frac{1}{T}
$$

The second integral gives

$$
\begin{aligned}
& \int_{0}^{T}\left[e^{-2 \gamma} \frac{\ln \left[(x(s)-y(s))^{2}+1\right]}{x(s)-y(s)}\right] d s \\
& \quad \leq e^{-2 \gamma} \frac{\ln \left[(\|x(s)-y(s)\|)^{2}+1\right]}{\|x(s)-y(s)\|} \times T .
\end{aligned}
$$

Using (102) and (103) in the R.H.S of (101), we get

$$
\leq\left(\frac{1}{T}\right)^{1 / 2}\left(e^{-2 \gamma} \frac{\ln \left[(d(x, y))^{2}+1\right]}{d(x, y)} \times T\right)^{1 / 2}
$$

Hence, from (99),

$$
d(T S x, T S y) \leq e^{-r} \sqrt{\frac{\ln \left[(d(x, y))^{2}+1\right]}{d(x, y)}} .
$$

Taking logarithms on both sides of (105) using $F_{1}(z)=$ $\ln (z)$ and the property of $F$, we get

$$
\ln (d(T S x, T S y)) \leq \ln \left(e^{-r} \sqrt{\frac{\ln \left[(d(x, y))^{2}+1\right]}{d(x, y)}}\right) .
$$

This implies that

$$
r+F(d(T S x, T S y)) \leq F\left(\sqrt{\frac{\ln \left[(d(x, y))^{2}+1\right]}{d(x, y)}}\right) .
$$

Let us choose a map $S x=\ln \left[(d(x, y))^{2}+1\right]$, it is obvious, $S \in T S$.

Thus, we conclude that for $x>y$ all conditions of Theorem 26 are satisfied. Hence, TS has a unique common fixed point $x^{*}$ which is the solution of integral equation (93).

\section{Data Availability}

This clause is not applicable to this paper.

\section{Additional Points}

Code Availability. There is no code required in this paper. Rights and Permissions. Open access. This article is distributed under the terms of the Creative Commons Attribution.

\section{Conflicts of Interest}

The authors declare that they have no competing interests.

\section{Authors' Contributions}

All authors contributed equally and significantly in writing this article. All authors read and approved the final manuscript.

\section{References}

[1] S. Banach, "Sur les opérations dans les ensembles abstraits et leur application aux équations intégrales," Fundamenta Mathematicae, vol. 3, pp. 133-181, 1922.

[2] R. Kannan, "Some results on fixed points," Bulletin of the Calcutta Mathematical Society, vol. 60, pp. 71-76, 1968.

[3] E. H. Connell, "Properties of fixed point spaces," Proceedings of the American Mathematical Society, vol. 10, no. 6, pp. 974979, 1959.

[4] V. Subrahmanyam, "Completeness and fixed-points," Monatshefte für Mathematik, vol. 80, no. 4, pp. 325-330, 1975.

[5] A. Branciari, "A fixed point theorem of Banach-Caccippoli type on a class of generalised metric spaces," Universitatis Debreceniensis, vol. 57, pp. 31-37, 2000.

[6] H. Aydi, C. M. Chen, and E. Karapinar, "Interpolative ĆirićReich-Rus type contractions via the Branciari distance," Mathematics, vol. 7, no. 1, p. 84, 2019.

[7] H. Aydi, E. Karapınar, and W. Shatanawi, "Tripled fixed point results in generalized metric spaces," Journal of Applied Mathematics, vol. 2012, Article ID 314279, 10 pages, 2012.

[8] B. Samet, "Discussion on a fixed point theorem of BanachCaccioppoli type on a class of generalized metric spaces," Publicationes Mathematiques, vol. 76, no. 4, pp. 493-494, 2010.

[9] I. R. Sarma, J. M. Rao, and S. S. Rao, "Contractions over generalized metric spaces," Journal of Nonlinear Sciences and Applications, vol. 2, no. 3, pp. 180-182, 2009.

[10] W. Shatanawi, A. al-Rawashdeh, H. Aydi, and H. K. Nashine, "On a fixed point for generalized contractions in generalized metric spaces," Abstract and Applied Analysis, vol. 2012, Article ID 246085, 13 pages, 2012.

[11] N. Souayah, H. Aydi, T. Abdeljawad, and N. Mlaiki, "Best proximity point theorems on rectangular metric spaces endowed with a graph," Axioms, vol. 8, no. 1, p. 17, 2019.

[12] A. Azam and M. Arshad, "Kannan fixed point theorem on generalized metric spaces," Journal of Nonlinear Sciences and Applications, vol. 1, no. 1, pp. 45-48, 2008.

[13] J. Munkres, Topology: Pearson New International Edition, Springer, Pearson, 2013.

[14] G. Buskes and A. Van Rooij, "Topological spaces," in Topological Spaces, pp. 187-201, Springer, New York, NY, 1997.

[15] R. C. Freiwald, An Introduction to Set Theory and Topology, Washington University in St. Louis, 2014.

[16] S. Moradi and D. Alimohammadi, "New extensions of Kannan fixed-point theorem on complete metric and generalized 
metric spaces," International Journal of Mathematical Analysis, vol. 47, no. 5, pp. 2313-2320, 2011.

[17] R. George, S. Radenovic, K. P. Reshma, and S. Shukla, "Rectangular b-metric space and contraction principles," Journal of Nonlinear Sciences and Applications, vol. 8, no. 6, pp. 10051013, 2015.

[18] A. Kari, M. Rossafi, E. M. Marhrani, and M. Aamri, "New fixed point theorems for $\theta-\varnothing$-contraction on rectangular $b$-metric spaces," Abstract and Applied Analysis, vol. 2020, Article ID 8833214, 12 pages, 2020.

[19] R. Malceski, A. Malceski, K. Anevska, and S. Malceski, “Common fixed points of Kannan and Chatterjea types of mappings in a complete metric space," Journal of Advances in Mathematics and Computer Science, vol. 2016, pp. 1-11, 2016.

[20] T. Rasham, G. Marino, and A. Shoaib, "Fixed points for a pair of F-dominated contractive mappings in rectangular b-metric spaces with graph," Mathematics, vol. 7, no. 10, p. 884, 2019.

[21] D. Wardowski, "Fixed points of a new type of contractive mappings in complete metric spaces," Fixed Point Theory and Applications, vol. 2012, no. 1, Article ID 94, 2012.

[22] N. Goswami, N. Haokip, and V. N. Mishra, "F-contractive type mappings in b-metric spaces and some related fixed point results," Fixed Point Theory and Applications, vol. 2019, no. 1, Article ID 13, 2019.

[23] A. Lukács and S. Kajántó, "Fixed point theorems for various types of $F$-contractions in complete $b$-metric spaces," Fixed Point Theory, vol. 19, no. 1, pp. 321-334, 2018.

[24] G. Minak, A. Helvaci, and I. Altun, "Ćirić type generalized F -contractions on complete metric spaces and fixed point results," Filomat, vol. 28, no. 6, pp. 1143-1151, 2014.

[25] H. Piri and P. Kumam, "Some fixed point theorems concerning F-contraction in complete metric spaces," Fixed Point Theory and Applications, vol. 2014, no. 1, Article ID 210, 2014.

[26] N. A. Secelean, "Weak F-contractions and some fixed point results," Bulletin of the Iranian Mathematical Society, vol. 42, no. 3, pp. 779-798, 2016.

[27] D. Wardowski and N. Van Dung, "Fixed points of F-weak contractions on complete metric spaces," Demonstratio Mathematica, vol. 47, no. 1, pp. 146-155, 2014.

[28] R. Batra, R. Gupta, and P. Sahni, "A new extension of Kannan contractions and related fixed point results," The Journal of Analysis, vol. 28, no. 4, pp. 1143-1154, 2020.

[29] D. Gopal, P. Kumam, and M. Abbas, Background and Recent Developments of Metric Fixed Point Theory, CRC Press, 2017.

[30] G. Jungck, "Compatible mappings and common fixed points," International Journal of Mathematics and Mathematical Sciences, vol. 9, no. 4, 779 pages, 1986.

[31] G. Jungck, "Commuting mappings and fixed points," The American Mathematical Monthly, vol. 83, no. 4, pp. 261-263, 1976.

[32] S. Sessa, "On a weak commutativity condition of mappings in fixed point considerations," Publications de l'Institut Mathématique, vol. 32, no. 46, pp. 149-153, 1982.

[33] H. Dasgupta, S. Chakrabarti, and P. Chakraborty, "A fixed point theorem of Caccioppoli-Kannan type on a class of generalized metric spaces," International Mathematical Forum, vol. 28, no. 9, pp. 1357-1361, 2014.

[34] H. K. Nashine, B. Samet, and C. Vetro, "Monotone generalized nonlinear contractions and fixed point theorems in ordered metric spaces," Mathematical and Computer Modelling, vol. 54, no. 1-2, pp. 712-720, 2011.
[35] J. J. Nieto and R. Rodríguez-López, “Contractive mapping theorems in partially ordered sets and applications to ordinary differential equations," Order, vol. 22, no. 3, pp. 223-239, 2005.

[36] A. Hussain and M. Adeel, "Remarks on "New fixed point theorems for $\alpha-H-\Theta$-contractions in ordered metric spaces"," Journal of Fixed Point Theory and Applications, vol. 21, no. 2, article 63, 2019.

[37] T. Kanwal, A. Hussain, H. Baghani, and M. de la Sen, "New fixed point theorems in orthogonal $F$-metric spaces with application to fractional differential equation," Symmetry, vol. 12 , no. 5 , p. $832,2020$.

[38] Z. Zhitao, "New fixed point theorems of mixed monotone operators and applications," Journal of Mathematical Analysis and Applications, vol. 204, no. 1, pp. 307-319, 1996.

[39] D. Baleanu, S. Rezapour, and H. Mohammadi, "Some existence results on nonlinear fractional differential equations," Philosophical Transactions of the Royal Society A: Mathematical, Physical and Engineering Sciences, vol. 371, no. 1990, p. 20120144, 2013.

[40] U. Zölzer, DAFX: Digital Audio Effects, 2002.

[41] A. A. Kilbas, H. M. Srivastava, and J. J. Trujillo, Theory and Applications of Fractional Differential Equations, Elsevier, 2006.

[42] L. Budhia, H. Aydi, A. H. Ansari, and D. Gopal, "Some new fixed point results in rectangular metric spaces with an application to fractional-order functional differential equations," Nonlinear Analysis: Modelling and Control, vol. 25, no. 4, pp. 580-597, 2020.

[43] Y. Zhou, J. Wang, and L. Zhang, Basic Theory of Fractional Differential Equations, World scientific, 2016.

[44] P. Borisut, P. Kumam, V. Gupta, and N. Mani, "Generalized $(\psi, \alpha, \beta)$-weak contractions for initial value problems," Journal of Fixed Point Theory and Applications, vol. 7, no. 3, p. 266, 2019.

[45] V. Gupta, W. Shatanawi, and N. Mani, "Fixed point theorems for $(\psi, \beta)$-Geraghty contraction type maps in ordered metric spaces and some applications to integral and ordinary differential equations," Journal of Fixed Point Theory and Applications, vol. 19, no. 2, pp. 1251-1267, 2017.

[46] J. Harjani and K. Sadarangani, "Generalized contractions in partially ordered metric spaces and applications to ordinary differential equations," Nonlinear Analysis: Theory, Methods Applications, vol. 72, no. 3-4, pp. 1188-1197, 2010.

[47] X. Li, A. Hussain, M. Adeel, and E. Savas, "Fixed point theorems for $Z_{\theta}$-contraction and applications to nonlinear integral equations," IEEE Access, vol. 7, pp. 120023-120029, 2019.

[48] F. Yan, Y. Su, and Q. Feng, "A new contraction mapping principle in partially ordered metric spaces and applications to ordinary differential equations," Journal of Fixed Point Theory and Applications, vol. 2012, no. 1, article 152, 2012.

[49] M. Shoaib, M. Sarwar, and T. Abdeljawad, "Hybrid fixed point theorem with applications to forced damped oscillations and infinite systems of fractional order differential equations," Journal of Function Spaces, vol. 2020, Article ID 4843908, 9 pages, 2020. 